Journal of Animal and Veterinary Advances 11 (11): 1812-1818, 2012

ISSN: $1680-5593$

(C) Medwell Journals, 2012

\title{
Trichostatin A Treatment on Two Types of Donor Cells for Somatic Cell Nuclear Transfer
}

\author{
${ }^{1,2}$ Ya-Xin Yao, ${ }^{2}$ Wei-Jun Guan, ${ }^{2}$ Xiang-Chen Li, ${ }^{2}$ Xiao-Hua Su, ${ }^{2}$ Yue-Hui Ma and ${ }^{1} \mathrm{He}-\mathrm{Ping} \mathrm{Li}$ \\ ${ }^{1}$ Northeast Forestry University, Harbin, 150040 Heilongjiang, China \\ ${ }^{2}$ Chinese Academy of Agricultural Sciences, Institute of Animal Science, 100193 Beijing, China
}

\begin{abstract}
An increased histone acetylation is associated with more effective formation of DNA replication complexes (Trichostatin A, TSA), a histone deacetylase inhibitor is able to enhance the pool of acetylated histone. Researchers observed the changes of the Sheep Fibroblasts Cells (SFCs) and Sheep Cumulus Cells (SCCs) being treated with TSA, a stronger immunofluorescent signal for acH4K12 was detected in the 1 cell stage $(\mathrm{p}<0.05)$ also the developmental rates of cloned embryos was increased significantly $(\mathrm{p}<0.05)$ however, a higher acetylation level were observed in SCCs naturely, no significant effects on acetylation level and developmental rates of cloned embryos were detected after TSA treatment. Moreover, the acetylation distribution in the group of SFC-TSA cloned embryos resembles that in In Vitro Fertilisation (IVF) embryos but TSA had no ability to modify the acetylation state of SCCs cloned embryos similar with the IVF embryos, only a hyperacetylate pattern was detected which means a proper pattern of acetylation in cloned embryo is more prone to be reprogrammed than hyperacetylation.
\end{abstract}

Key words: TSA, histone acetylation, donor cell, reprogramming, IVF, China

\section{INTRODUCTION}

An abnormal reprogramming of epigenetic state led to the inefficiency of somatic cell cloning (William et al., 2001; Hochedlinger and Jaenisch, 2006). The completely reprogramming of a donor nucleus is up to the donor cells' epigenetic state (Enright et al., 2003; Shi et al., 2003) and during normal development, early embryos undergo a well-orchestrated series of DNA methylation and histone modification changes that are believed to play an important role in establishing a chromatin state permissive to early embryonic gene expression.

Histone acetylation is heritable epigenetic modifications thus, the histone code may function as an epigenetic marker that is directly associated with transcriptional activation (Kurdistani and Grunstein, 2003; Turner, 2002; Vogelauer et al., 2002). It has been supposed that increased histone acetylation levels on most amino acid residues leads to looser binding of the nucleosome to DNA as a result of which the chromatin becomes looser in structure and then enters a transcriptionally permissive state (Zlatanova et al., 2000). Moreover, increased histone acetylation is associated with more effective formation of DNA replication complexes (Vogelauer et al., 2002). There are more contributing factors in the development of reconstructed embryos which could be improved if to donor cells were treated properly.
As is known, TSA, a histone deacetylase inhibitor is able to enhance the pool of acetylated histone (Kishigami et al., 2006). Treatment of donor cells with chromatin modifying agents may improve their ability to be reprogrammed. It has been shown that cumulus cells and fibroblasts are more prone to be reprogrammed than other types (Wakayama et al., 1998). Moreover, fibroblasts and cumulus cells of the domestic cat have an inherently long $\mathrm{GO} / \mathrm{Gl}$ phase and it has been shown that cumulus cells and fetal fibroblasts are more ready to be reprogrammed and were consequently chosen as donor cells.

Although, how the epigenetic state is maintained and stably inherited throughout preimplantation development is unclear, the histone acetylation patterns of cloned embryos is aberrant. So, researchers assessed whether the aberrant histone acetylation patterns of cloned embryos could be corrected by the treatment of donor cells with TSA.

Chromatin immunoprecipitation experiments have shown that histone $\mathrm{H} 4$ is hyperacetylated in the promoter regions of active genes selectively recognizes and associates with $\mathrm{AcH} 4 \mathrm{~K} 12$ (lysine 12; H4K12) to amplify transcription (Kanno et al., 2004). So, researchers used an anti-acetyl-histone $\mathrm{H} 4 \mathrm{~K} 12$ to detect the acetylation level and to reveal the process of epigenetic reprogramming. Staining pattern has been examined in spare sheep embryos. That is from zygotes up to the blastocyst stage.

Corresponding Author: He-Ping Li, Northeast Forestry University, Harbin, 150040 Heilongiiang, China 
Accordingly, in this research we observed the changes of the SFCs and SCCs upon treatment with TSA, through cell cycle, acetylation level and the developmental rates of cloned embryos.

\section{MATERIALS AND METHODS}

Preparation of donor cells: Fibroblast cells were routinely cultured according to the methods described by Li et al. (2009). The cumulus cells were isolated from the Cumulus-Oocyte Complexes (COCs) in Synthetic Oviductal Fluid-Hepes (SOF-Hepes) with hyaluronidase ( $1 \mathrm{mg} \mathrm{mL}^{-1}$ ). The cells were collected into $1.5 \mathrm{~mL}$ tubes, centrifuged at $500 \mathrm{~g}$ for $5 \mathrm{~min}$ and then resuspended in DMEM supplemented with 10\% FBS. The cell suspension was transferred to petri dishes and cultured for 2-3 days at $38.5^{\circ} \mathrm{C}$ with $5 \%$ humidified $\mathrm{CO}_{2}$.

TSA treatment: TSA dissovled in dimethylsulfoxide was diluted in media to yield solutions of different concentrations and the suitable handling time and concentration was discussed in the previous study (Koo et al., 2002). SFCs and SCCs of passage 2-4 in logarithmic phase were incubated in the culture media containing $10 \mathrm{ng} \mathrm{mL} \mathrm{mL}^{-1} \mathrm{TSA}$ for $24 \mathrm{~h}$.

In Vitro Fertilisation (IVF): Oocytes were washed three times inmodified Tris-buffered medium (IVF medium). Ten to fifteen oocytes were transferred into a $45 \mathrm{~mL}$ droplet of IVF medium overlaid with paraffin oil. Fresh semen collected from boars at an artificial insemination centre was centrifuged twice at $1500 \mathrm{~g}$ for $5 \mathrm{~min}$. The spermatozoa were resuspended in IVF medium at the concentration of $1.0 \times 10^{6}$ cells $\mathrm{mL}^{-1}$. Finally, $5 \mathrm{~mL}$ of the sperm suspension was added to each droplet and cultured in a humidified atmosphere with $5 \% \mathrm{CO}_{2}$ in air at $39.8^{\circ} \mathrm{C}$ for $6 \mathrm{~h}$.

Preparation of recipient oocytes and SCNT: The oocytes were matured in vitro as previously described (Li et al., 2011). All mature oocytes having an extruded first polar body with homogeneous cytoplasm were used for nuclear transfer experiments.

Routine procedure of SCNT was described in detail by Wee et al. (2006). The NT embryos were cultured in $20 \mu \mathrm{L}$ medium drops and individually in $3 \mathrm{~mL}$ drops of SOF with $4 \mathrm{mg} \mathrm{mL}^{-1} \mathrm{BSA}$ in a humidified atmosphere with $5 \% \mathrm{CO}_{2}$ at $38.5^{\circ} \mathrm{C}$.

Detection of acH4K12 immunofluorescence IVF and cloned embryos: All staining procedures were performed at room temperature in $10 \mathrm{mM}$ isotonic PBS supplemented with $1 \%$ BSA. The IVF and cloned embryos in groups of 5 for 3 independent repeats each were fixed for $1 \mathrm{~h}$ in freshly-prepared 4\% paraformaldehyde in PBS without BSA. They were then washed and permeabilized for 20 min with $0.5 \%$ Triton $\mathrm{X}-100$, washed again for $20 \mathrm{~min}$ and incubated with the primary anti-acetyl antibodies: anti-acetyl-histone $\mathrm{H} 4 \mathrm{~K} 12$ at $2 \mu \mathrm{g} \mathrm{mL}^{-1}$ (1:200 in stock; Santa Cruz Biochemistry) for $2 \mathrm{~h}$. After incubation and being washed for $20 \mathrm{~min}$, the embryos were incubated with FITC conjugated mouse anti rabbit secondary antibodies at $4 \mu \mathrm{g} \mathrm{mL} \mathrm{m}^{-1}(1: 100$ in stock; Santa Cruz Biochemistry) for $1 \mathrm{~h}$. They were then washed and mounted for observation with PI containing mounting medium. Finally, the samples were mounted between a coverslip and a glass slide supported by 4 columns of a mixture of petroleum jelly and paraffin $(9: 1, \mathrm{vol} / \mathrm{vol})$.

Slides were scanned by using a confocal laserscanning microscope (Nikon TE-2000-E, Japan) with an argon-krypton laser at 488 and $563 \mathrm{~nm}$ and 2-channel scanning for detection of FITC and PI, respectively. Exposure time of exciting lights was uniform for respective channels.

The nuclear intensities of integrated fluorescence were measured by manually outlining all nuclei, 20 nuclei per morula and 30 nuclei per blastocyst (Suteevun et al., 2006). The total fluorescence intensity emitted by each nucleus was measured on antibody stained images after background subtraction by Image J Software (image processing and analysis in Java, http://rsb.info.nih.gov/ij/) and was averaged per embryo (Carmona et al., 2007; Mitalipov et al., 2002).

The result of immunofluorescence was observed under a laser scanning confocal microscope (Nikon Inc., Japan) and the intensities of fluorescences were determined using an image analyzer system, EZ-C1 3.7 Free Viewer (Nikon Inc., Japan) by the fluorescence signal ratios of acH4K12 to $\mathrm{PI}$.

Statistical analysis: Three repetitions were performed for each group. The experimental datas were analysed using Duncan's multiple range tests using the General Linear Model procedure in SPSS Software (SPSS Inc., Chicago, IL). A paired t-test was used to compare the values of different immunofluorescence intensities. A value of $\mathrm{p}<0.05$ was considered to be statistically significant.

\section{RESULTS AND DISCUSSION}

Histone acetylation status of donor cells transferred into non-activated oocytes: After enucleation, donor cells were individually inserted into the perivitelline space of single enucleated oocytes then fused but not activated. 

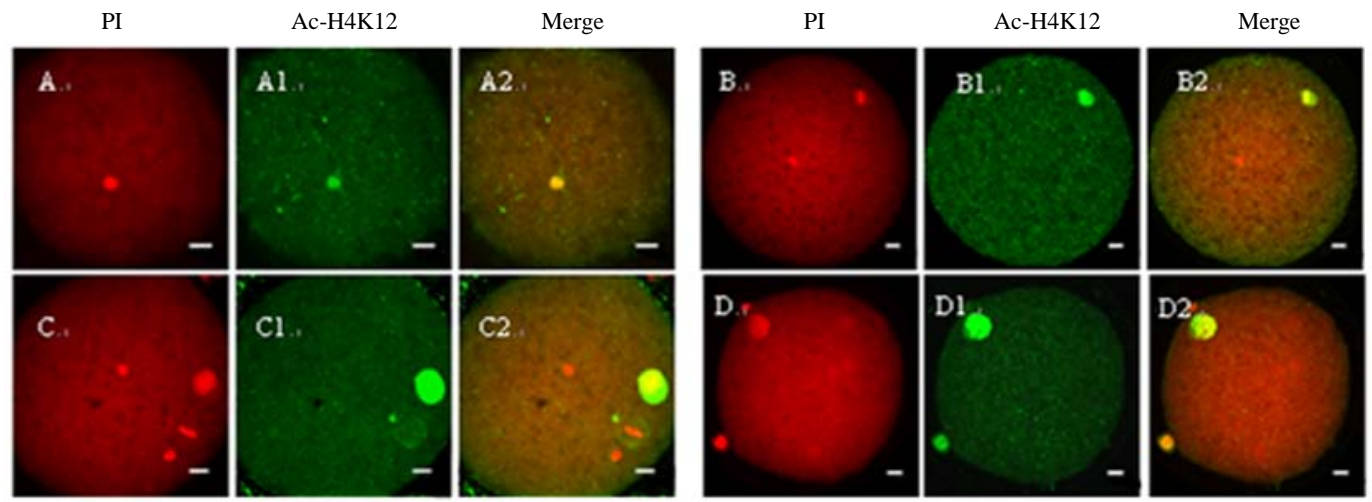

Fig. 1: The immunofluorescent staining of donor cell acetylation transferred into non-activated oocytes, A: The immunofluorescent staining of SFC acetylation, $\mathrm{PI}$, red; $\mathrm{A}_{1}$ : anti-H4K12 acetyl immunofluorescent, Green; $\mathrm{A}_{2}$ : Merge, the same as below; B: TSA treated SFC; C: the immunofluorescent staining of SCC acetylation, D: TSA treated SCC. Scale; Bar $=20 \mu \mathrm{m}$

Researchers got the non-activated oocytes after the fused oocytes cultured in the SOF for $1 \mathrm{~h}$. Then, we obtained a similar conclusion with the histone acetylation status of SFCs (Fig. 1 and 2). Surprisingly, in the SCCs group, to the contrary of a strong immunofluorescence signal detected in SCCs TSA-treated oocytes, there were nearly no change in histone acethylation of control embryos in another words, a significant higher level of histone acetylation was only found in the TSA-treated SCC derived oocytes rather than control (Fig. 2).

Histone acetylation patterns of IVF embryos and cloned embryos: For successful development of cloned embryos, epigenetic status of donor cells should be reprogrammed to that of normal embryos. It was therefore, investitgated whether the histone acetylation patterns of cloned embryos could be corrected by the treatment of donor cells with TSA and the results suggested the patterns were just like those in IVF embryos. Consequently, the acetylation state in SFC-TSA cloned embryos were resemble that in IVF embryos, suggesting that TSA had the ability to modify the acetylation pattern of SCNT embryos and reprogramming of this epigenetic mark was aberrant in cloned embryos and could be corrected by the treatment of donor cells with TSA (Fig. 3 and 4).

The cloned embryos from SFC-TSA cells showed a similar acetylation pattern as IVF embryos. While SFCcloned embryos, moderate acetylation levels were maintained at all stages, there is no significant fluctuate which is bad for the embryos development. This explained why the development rate of TSA-treated SFC derived embryos was close to that of the IVF embryos.
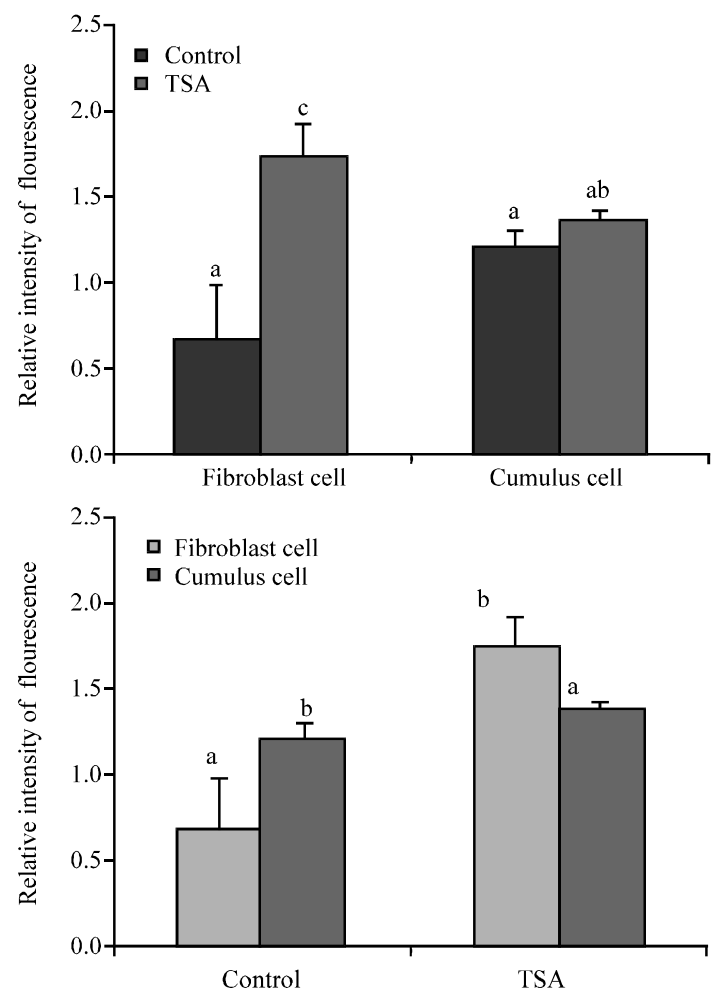

Fig. 2: The quantitative analysis of acH4K12 signals in the donor cell transferred into non-activated oocytes. Each value is formulated as mean \pm SEM. Different letters means significant difference among the treatments $(\mathrm{p}<0.05)$ while the same letter means non-significant difference among the treatments $(\mathrm{p}>0.05)$

An intensified signal was detected and a much stronger staining was observed in TSA-treated cloned 


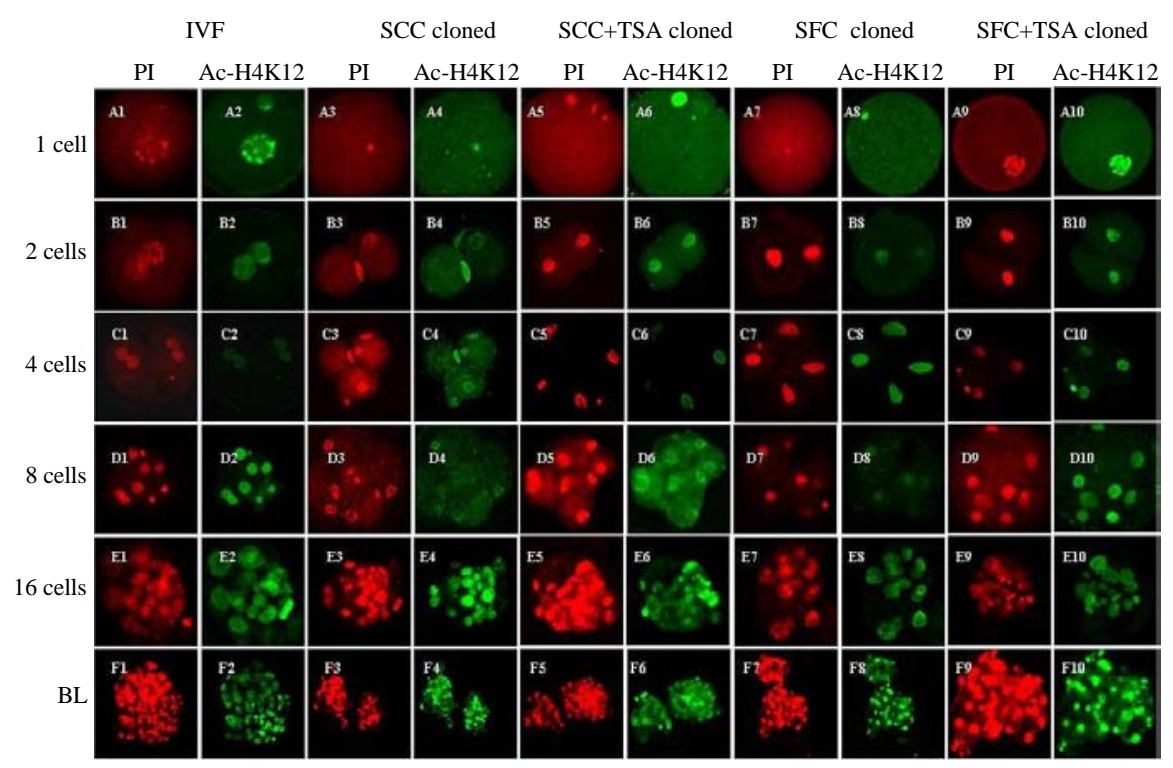

Fig. 3: The immunofluorescent stainingof IVF and cloned embryo acetylation. IVF: 1 cell 13/15; 2 cell 15/18; 4 cell 8/10; 8 cell 13/13; 16 cell 9/9; blastocysts 8/9; SFC: 1 cell $1010 ; 2$ cell 6/8, 4 cell 10/10, 8 cell $7 / 9 ; 16$ cell 8/10; blastocysts 8/8; SFC+TSA: 1 cell 15/15, 2 cell 10/11, 4 cell 10/10, 8 cell 9/10, 16 cell 9/10, blastocysts $6 / 7$; SCC: 1 cell 11/13; 2 cell $7 / 9 ; 4$ cell 11/11; 8 cell 8/9, 16 cell 9/11; blastocysts $7 / 7$; SCC+TSA: 1 cell 12/15, 2 cell 12/13, 4 cell 11/12; 8 cell 10/10; 16 cell 9/11 and blastocysts 6/6) IVF: in vivo fertilized embryos. SCF and SFC+TSA, SCC, SCC+TSA embryos cloned from, sheep fibroblast cells, TSA-treated sheep fibroblast cells, sheep cumulus cells, TSA-treated sheep cumulus cells respectively; BL: Blastocysts. Cells were immunostained with anti-acH4K12 (green) and counterstained with PI for chromatin (red). The images represent acH4K12 patterns as in the majority of stained embryos. All images are at the magnification x 200
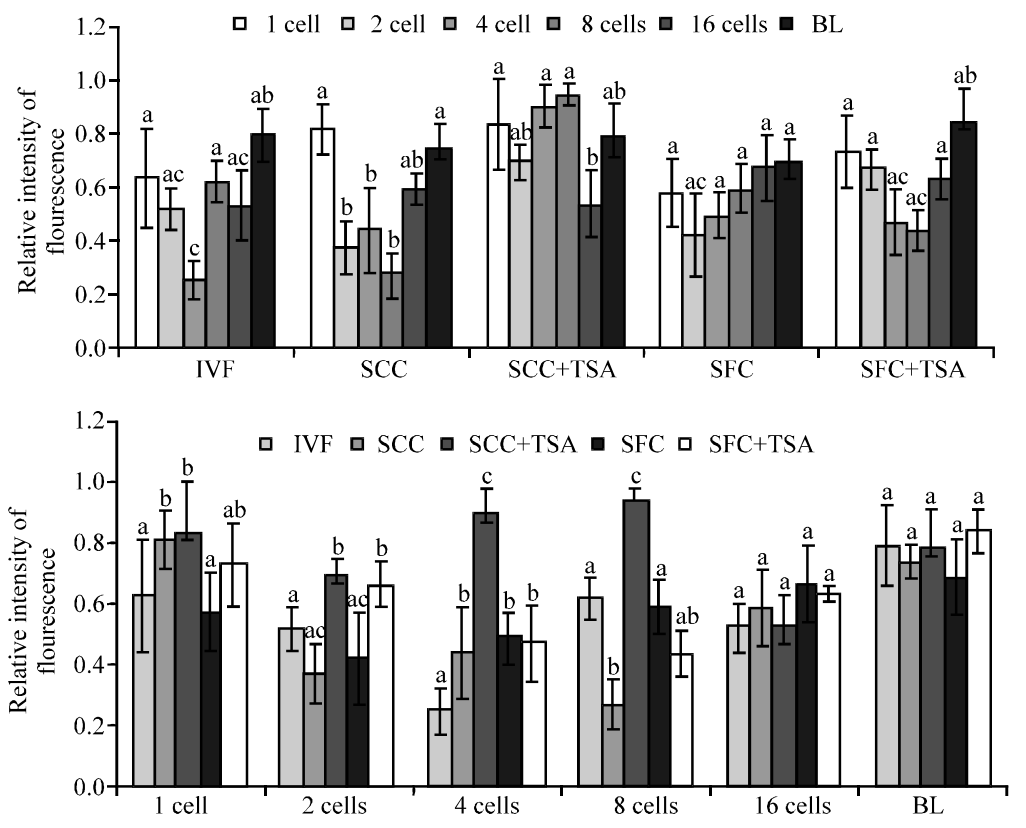

Fig. 4: The quantitative analysis acH4K12 signals of IVF and cloned embryos. Each value is formulated as mean \pm SEM. Different letters means significant difference among the treatments $(\mathrm{p}<0.05)$ while same letter means nonsignificant difference among treatments $(\mathrm{p}>0.5)$ 
embryos than in the normal cloned embryos and especially in the SCC-derived normal cloned embryos, it displayed low levels of acH4k12 at 2-8 cells stages which increases to moderate levels at the 16 cells stages and blastocyst stages however, in embryos cloned from SCC-TSA oocytes, there were hyperacetylated at all stages including 2-8 cells periods (Fig. 4).

The acetylation patterns in SCC-TSA cloned embryos indicated that the hyperacetylation was not suitable for the cloned embryos which would reduce the developmental rates.

Development of cloned embryos and IVF embryos: After $\mathrm{SCNT}$, in vitro developmental capacity of cloned sheep embryos with differently treated donors was investigated (Table 1). In the SFCs groups, it was suggested there was a significant increase $(\mathrm{p}<0.05)$ in developmental rates of TSA-treated SFCs NT embryos. While the TSA-treated SFCs as donor cells was not to improve the development of the NT embryos, on the opposite however, to reduce it but not significantly, indicating that TSA might not be suitable to treat the cumulus cells.

Considering the results showed above, researchers compared the developmental capactity of NT embryos with TSA-treated cells with that of IVF embryos (Table 1). The developmental rate of NT embryos with TSA-treated SFCs was close to that of the IVF embryos (28.2 vs. $32.8 \%$ ).

The acetylation of nuclear histones is thought to play key roles in the propagation of genomic function information from one cell generation to the next by retaining this information during the mitotic phase (Turner, 2002). Proper patterns of histone acetylation which were characterized by specific chromatin modifications, play a key role in nuclear reprogramming after SCNT and can determine the efficiency of SCNT.

According to this study, the histone acetylation patterns of cloned embryos are aberrant. Firstly there was

Table 1: Development of IVF embryos and cloned embryos from different types of somatic donor cells

\begin{tabular}{lccc}
\hline Embryo type & No. of embryos & No. of cleaved & No. of blastocysts \\
\hline IVF & 265 & $190(71.8 \pm 5.1)^{\mathrm{b}}$ & $87(32.8 \pm 4.5)^{\mathrm{c}}$ \\
SFC & 293 & $168(57.3 \pm 8.9)^{\mathrm{a}}$ & $21(7.1 \pm 7.6)^{\mathrm{a}}$ \\
SFC+TSA & 289 & $234(81.2 \pm 3.4)^{\mathrm{c}}$ & $81(28.2 \pm 6.3)^{\mathrm{bc}}$ \\
SCC & 288 & $203(70.5 \pm 6.7)^{\mathrm{b}}$ & $56(19.4 \pm 8.3)^{\mathrm{b}}$ \\
SCC+TSA & 274 & $178(64.9 \pm 3.8)^{\mathrm{ab}}$ & $37(13.5 \pm 8.9)^{\mathrm{ab}}$ \\
\hline SFC: Sheep Fibroblast Cells, SCCs: Sheep Cumulus Cells, SD: Standard \\
Deviation, SFC+TSA: SFC treated with 10 ng mL m $^{-1}$ TSA, SCC+TSA: \\
SCC treated with 10 ng mL ${ }^{-1}$ TSA. ${ }^{\mathrm{a}-c}$ Values with different superscripts in \\
the same column are significantly different. The cleavage rates and the \\
blastocyst rates were determined at $48 \mathrm{~h}$ and 7 days after activation, \\
respectively
\end{tabular}

a moderate acetylation level at the 1 cell stage, secondly deactylation in the 2,4 or 8 cells stage and high level of histone acetylation was detected till the 16 cells stage and blastocyst stage. In a recent study, the deactylationreacetylation transformation occurred in both experiments as well as in other species (Wee et al., 2006) and insofar all species shared the erase and rebuild pattern in normal embryo development (Suteevun et al., 2006). In the IVF, SCC cloned, SFC+TSA cloned embryos which exhibited more similarities in acH4K12 patterns to the IVF embryos, decreased developmental rates and low acetylation levels were detected at 4 or 8 cells stage indicating that low acetylation level was correlated with the embryos development. Genomic activation in NT or even the TSA-NT embryo is insufficient for subsequent embryonic development because of its low level of histone H4 acetylation at 8 cells stage (Carmona et al., 2007). Abnormal DNA methylation and histone acetylation obtained in previous studies which perhaps subsequently prevent the correct expression of crucial genes at 8 cells stage might explain the developmental loss observed in this study at this phase and in a previous report by Mitalipov et al. (2002).

The methods of treating donor cells were possibly suitable for one type but not for another. The treatment of SFCs with TSA increased the proportion of NT embryos developing to blastocysts ( 28.2 vs. $7.1 \%$ of non-treated SFCs). However, the TSA-treated SCCs NT embryos were not so fortunate in terms of decreased of developmental rates (13.5 vs. 19.4\% with non-treated SCCs). It was found in this study that the levels of histone acetylation in the SCCs are higher than those in the SFCs and it might be the epigenetic modifications in SCCs that facilitated the reprogramming. TSA induces nuclear histone hyperacetylation which would make corresponding genes overexpressed. There might be some genes harmful to reprogramming that should be silent were expressed in this stage which prevented the embryos from further development. On the other hand, researchers have recognized that the cumulus cells were relatively difficult to culture in long term compared with the fibroblasts and those from most individuals could only be subcultured to passage 10 at most but cell lines from a few individuals had no comparable in vitro proliferative potential and their morphology is apt to change (Yang et al., 2007) the cumulus cells were might be so fragile that couldn't endure the treatment.

Although, the developmental rates of the cloned embryos are quite different, each of the cloned embryos to the blastocyst stage has the same high level of histone 
acetylation (Fig. 4), either in the TSA-treated group or those non-treated. It is reported that increased histone acetylation is associated with the more effective nucleus reprogramming (Cervera and Garcia-Ximenez, 2003). An interesting discovery from Smith suggested that global gene expression profile of bovine SCNT embryos is similar to that of embryos produced in vivo, suggesting that by the blastocyst stage, the SCNT embryos have undergone a relatively complete and correct nuclear reprogramming at molecular level (Smith et al., 2005).

\section{CONCLUSION}

This research yield important information regarding mechanisms responsible for nuclear reprogramming. However, rather than that all the reprogramming events that should take place in a successful NT are apparent throughout the relatively short embryo culture period and it is possible that they will happen in the fetal survival following embryo transfer during the days of gestation. A key mystery that remains to be unvailed is how reprogramming events happen after embryo transplantation.

\section{ACKNOWLEDGEMENTS}

The research was supported by the National Key Technology R and D Program (2006BAD13B08, 2007AA10Z170), the Ministry of Agriculture of China for transgenic research (2008ZX08009-003) and the 863 National Major Research Program (2007AA10Z170). Central Public-interest Scientific Institution Basal Research Fund (2010jc-10). Ya-Xin Yao and Wei-Jun Guan contributed equally to this research.

\section{REFERENCES}

Carmona, R., D. Macias, J.A. Guadix, V. Portillo, J.M. Perez-Pomares and R. Munoz-Chapuli, 2007. A simple technique of image analysis for specific nuclear immunolocalization of proteins. J. Microsc., 225: 96-99.

Cervera, R.P. and F. Garcia-Ximenez, 2003. Oocyte age nuclear donor cell type affect the technical efficiency of somatic cloning in rabbits. Zygote, 11: 151-158.

Enright, B., B. Jeong, X. Yang and X. Tian 2003. Epigenetic characteristics of bovine donor cells for nuclear transfer: Levels of histone acetylation. Biol. Reprod., 69: 1525-1530.
Hochedlinger, K. and R. Jaenisch, 2006. Nuclear reprogramming and pluripotency. Nature, 441: 1061-1067.

Kanno, T., Y. Kanno, R.M. Siegel, M.K. Jang, M.J. Lenardo and K. Ozato, 2004. Selective recognition of acetylated histones by bromodomain proteins visualized in living cells. Mol. Cell., 13: $33-43$.

Kishigami, S., E. Mizutani, H. Ohta, T. Hikichi and N.V. Thuan et al., 2006. Significant improvement of mouse cloning technique by treatment with trichostatin A after somatic nuclear transfer. Biochem. Biophys. Res. Commun., 340: 183-189.

Koo, D.B., Y.K. Kang, Y.H. Choi, J.S. Park and H.N. Kim et al., 2002. Aberrant allocations of inner cell mass and trophectoderm cells in bovine nuclear transfer blastocysts. Biol. Reprod., 67: 487-492.

Kurdistani, S.K. and M. Grunstein, 2003. Histone acetylation and deacetylation in yeast. Nat. Rev. Mol. Cell. Biol., 4: 276-284.

Li, X.C., H. Yua, C.Y. Li, X.H. He and Q.J. Zhao et al., 2009. Establishment and characterization of a fibroblast cell line derived from Jining Black Grey goat for genetic conservation. Small Rumin. Res., 87: 17-26.

Li, X.C., Y.X. Yao, P. Liu, D.P. Jin, W.J. Guan and Y.H. Ma, 2011. The apoptotic effects of alcohol shock on bovine oocytes. J. Anim. Vet. Adv., 10: 2679-2682.

Mitalipov, S.M., R.R. Yeoman, K.D. Nusser and D.P. Wolf, 2002. Rhesus monkey embryos produced by nuclear transfer from embryonic blastomeres or somatic cells. Biol. Reprod., 66: 1367-1373.

Shi, W., A. Hoeflich, H. Flaswinkel, M. Stojkovic, E. Wolf and V. Zakhartchenko, 2003. Induction of a senescent-like phenotype does not confer the ability of bovine immortal cells to support the development of nuclear transfer embryos. Biol. Reprod., 69: 301-309.

Smith, S.L., R.E. Everts, X.C. Tian, F. Du and L.Y. Sung et al., 2005. Global gene expression profiles reveal significant nuclear reprogramming by the blastocyst stage after cloning. Proc. Natl. Acad. Sci. USA., 102: 17582-17587.

Suteevun, T., R. Parnpai, S.L. Smith, C.C. Chang, S. Muenthaisong and X.C. Tian, 2006. Epigenetic characteristics of cloned and in vitro-fertilized Swamp Buffalo (Bubalus bubalis) embryos. J. Anim. Sci., 84: 2065-2071.

Turner, B.M., 2002. Cellular memory and the histone code. Cell, 111: 285-291. 
Vogelauer, M., L. Rubbi, I. Lucas, B.J. Brewer and M. Grunstein, 2002. Histone acetylation regulates the time of replication origin firing. Mol. Cell., 10: 1223-1233.

Wakayama, T., A.C. Perry, M. Zuccotti, K.R. Johnson and R. Yanagimachi, 1998. Full-term development of mice from enucleated oocytes injected with cumulus cell nuclei. Nature, 394: 369-374.

Wee, G., D.B. Koo, B.S. Song, J.S. Kim and M.J. Kang et al., 2006. Inheritable histone H4 acetylation of somatic chromatins in cloned embryos. J. Biol. Chem., 281: 6048-6057.
William, M.R., E. Kevin and J. Rudolf, 2001. Nuclear cloning and epigenetic reprogramming of the genome. Science, 293: 1093-1098.

Yang, F., R. Hao, B. Kessler, G. Brem, E. Wolf and V. Zakhartchenko, 2007. Rabbit somatic cell cloning: Effects of donor cell type histoneacetylation status and chimeric embryo complementation. Soc. Reprod. Fertil., 133: 119-230.

Zlatanova, J., P. Caiafa and H.K. Van, 2000. Linker histone binding and displacement: Versatile mechanism for transcriptional regulation. FASEB J., 14: 1697-1704. 